Songkla, Thailand; ${ }^{3}$ Mahasaraklam University, Mahasaraklam, Thailand; ${ }^{4}$ Chiangmai University, Chiangmai, Thailand; ${ }^{5}$ Department of Nursing, Faculty of Medicine, Ramathibodi Hospital, Mahidol University, Bangkok, Thailand

Introduction This study aims to test the casual model of gender, family atmosphere, financial status, resilience, and risk-taking behaviour of Thai adolescents. The study also examines the direct and indirect effects among variables.

Methods The sample for this study consisted of 2715 adolescents studying at secondary schools in four regions (the central, southern, North, and Northeast) of Thailand during January to March, 2009. The model was tested using the LISREL program.

Results Data analysis shows that the proposed model was fit with the empirical data well $\left(\chi^{2}(7)=12.158, p=0.095 ; \mathrm{GFI}=0.998\right.$; $\mathrm{AGFI}=0.996$; RMSEA=0.017). When considering the influence of the study variables, the results demonstrated as follows: (1) Male reported being resilient less than females and engaging in risk-taking behaviour more than females ( $p<0.01)$; (2) The financial status had the positively direct effect on resilience and the negatively direct effect on risk-taking behaviour. The financial status also had the indirect effects on risk-taking behaviours through resilience $(p<0.01)$; (3). The family atmosphere had the positively direct effect on resilience but had the indirect effects on the risk-taking behaviour through resilience $(p<0.01)$; and (4) The resilience had the negatively direct effect on the risk-taking behaviour $(p<0.01)$.

Conclusion The information from this study could be applied to develop the programs to promote resilience for adolescents to prevent risk-taking behaviour.

\section{P2-460 PROPOSED METHOD FOR EVALUATION OF FRAILTY}

doi:10.1136/jech.2011.142976l.88

${ }^{1} \mathrm{D}$ P Nunes, ${ }^{1} \mathrm{~T}$ da Silva Alexandre, ${ }^{1} \mathrm{~L}$ P Corona, ${ }^{2} \mathrm{~J}$ L Santos, ${ }^{3} Y$ A 0 Duarte, ${ }^{*}$ ${ }^{1} \mathrm{M}$ L Lebrao. ${ }^{1}$ Department of Epidemiology, University of Sao Paulo, São Paulo, São Paulo, Brazil; ${ }^{2}$ Department of Social Medicine, University of Sao Paulo, São Paulo, São Paulo, Brazil; ${ }^{3}$ Department of Nursing Medical Surgery, University of Sao Paulo, Sao Paulo, São Paulo, Brazil

Introduction The evaluation of frailty requires some measurable components. In our environment, it is known that the use of these measures on a large scale will not easily operationalise. Given this, we study the possibility of identifying the syndrome of frailty through subjective questions.

Objective To validate the subjective components for evaluation of frailty.

Method This study is part of the SABE Study - Health, Well-being and Ageing, held in São Paulo, Brazil. This analysis used 433 elderly (age $\geq 75$ years) in 2009 . We adopted the phenotype of frailty proposed by Fried et al, as a gold standard (objective evaluation of unintentional weight loss, fatigue reported, reduced grip strength, reduced walking speed and low physical activity). In this model, elderly with three or more components are considered frail; those with one or two are pre-frail. Subjective evaluation was performed using dichotomous questions for each component. We calculated the reliability, sensitivity, specificity and positive and negative predictive values.

Results The subjective evaluation of an instrument is valid and reliable. The sensitivity was $89.7 \%$ among pre-frail and $63.2 \%$ among the frail. By analysing frailty process (pre-frail+frail) the sensitivity was $90 \%$, positive predictive value was $85.2 \%$ and negative predictive value was $32.7 \%$.

Conclusion The subjective evaluation of frailty is a good tool to identify frailty process in elderly.

\section{P2-461 WOMEN VULNERABILITY TO HIV/AIDS IN THE EYE OF CULTURE: A CASE STUDY OF ANAMBRA STATE IN NIGERIA}

doi:10.1136/jech.2011.142976l.89

${ }^{1} \mathrm{C}$ Okonkwo, ${ }^{* 1}$ I Oguadimma, ${ }^{1} \mathrm{P}$ Umeojiakor, ${ }^{1} \mathrm{~N}$ Ezekwueche. ${ }^{1}$ Youth Network on HIVI AIDS Population \& Development in Nigeria, Awka, Anambra, Nigeria; ${ }^{2}$ Hope Givers Organisation, Awka, Anambra, Nigeria

Background Culture is people way of life but some cultures are barbaric that they contain ingredients that relegate women to the background and the situation is worsening with HIV infection. The aspect that requires urgent attention is that majority of women involved think that these cultural practices are normal thus; do not perceive any risk to HIV infection. This work was to evaluate the knowledge of rural women on sexual violence, mode of HIV transmission (cultural perspective) and predisposing cultural practices in Anambra State rural communities.

Methodology Questionnaires and In-depth Interview (IDIs) survey involved in the selection of 150 respondents through multistage technique. This structured questionnaires covered areas such as knowledge of violence, HIV/AIDS and cultural practices that make one vulnerable to HIV infection in the area.

Result All the respondents have heard of HIV/AIDS but $78 \%$ of them do not know all the modes of HIV transmission. Study further revealed that $55 \%$ of women were victims of some type of sexual coercion with a husband in their lifetime, 35\% with men other than their husband, all openly admit to physical violence and that sexual violence is highly underreported. Also, all agreed to practices that helps keep their husbands or their own family linage alive.

Conclusion There is serious need for programmers to step up efforts towards addressing reproductive health and rights of women especially widows and families without child/children otherwise the aim of impact mitigation of HIV/AIDS among women of reproductive age in the selected rural communities will be defeated.

\section{P2-462 THE INFLUENCE OF DISTRESS ON DISABILITY, PHYSICAL ACTIVITY AND PAIN INTENSITY AFTER 7 DAYS OF ACUTE SEVERE LOW BACK PAIN}

doi:10.1136/jech.2011.142976l.90

${ }^{1} \mathrm{P}$ Olaya-Contreras, ${ }^{*}{ }^{1} \mathrm{~J}$ Styf, ${ }^{2} \mathrm{M}$ Olsson, ${ }^{1} \mathrm{~A}$ Kaigle-Holm, ${ }^{1} \mathrm{~T}$ Hansson. ${ }^{1}$ Department of Orthopaedics, University of Gothenburg, Sahlgrenska University Hospital, Gothenburg, Sweden; ${ }^{2}$ Department of Statistics, Chalmers University, Gothenburg, Sweden

Low back pain (LBP) is described as highly recurrent and frequently persistent public health worldwide. The "stay active" concept has been regarded as the most appropriate treatment recommendation for patients with acute LBP. The objective of this study was to evaluate the influence of distress on disability, physical activity and pain intensity in subjects with severe acute LBP.

Methods A Randomised Control Trial (RCT) was conducted, 99 employed subjects (mean age 45 years, 20-63), 61\% white- and 39\% blue-collar workers with acute LBP were examined within $48 \mathrm{~h}$ after the onset of pain. All patients were initially assessed using the Depression Anxiety and Positive Outlook Scale (DAPOS) and the Tampa Scale of Kinesiophobia (TSK) questionnaires. Thereafter, the patients documented the following in a diary over a 7-day period: pain intensity, disability rating index (DRI) and step count (pedometer). Linear Mixed Models (LMM) for repeated measures were employed for the statistical analyses. All results were adjusted for age, gender, treatment, number of days and for the interaction term (treatment *DAPOS-D).

Results Prospectively, DRI and pain intensity responses were differentially mediated by the treatment, in interaction with the 
scores of DAPOS $(p<0.05)$. Patients with high scores on DAPOS exhibited higher risk for worse pain-disability after follow-up. Additionally, patients with higher baseline scores on TSK $(>38)$ had a lower step count over time $(\mathrm{p}<0.05)$.

Conclusion Depressed mood and fear of movement affect the outcomes of disability, the level of physical activity and the pain intensity in patients with acute LBP.

\section{P2-463 USE OF SUBSTANCE AMONG RESIDENTS OF KARACHI: REASONS AND COSTS OF USING SUBSTANCES}

doi:10.1136/jech.2011.142976l.91

M Parveen, ${ }^{*}$ S Pyar Ali. Aga Khan University, Sindh, Pakistan

Introduction Use of substances (pan, chaalia, ghutka, niswar) is associated with serious health risks. In Pakistan most people use substances from early years of life which keeps them on risk of short life span. The aim of this study was to estimate the costs of using these substances and to understand the reasons for using substances. Methodology This was a cross-sectional study conducted in two residential colonies in Karachi, Pakistan during 2008-2009. Pre-coded structured questionnaire was administered to collect the data on socio demographics, use of substance, costs and reasons for use of substances. Results From 124 randomly selected residents, 107 (86\%) agreed to respond. All the selected participants were between the ages $10-71$ years (mean $\pm S D$ age $36.2 \pm 16.4$ ). Daily use of substances was significantly higher among males $(p<0.001)$. Further, the use was higher among adolescents than adults $(p<0.001)$ and interestingly less educated consumed less than high educated $(p=0.06)$. Males are

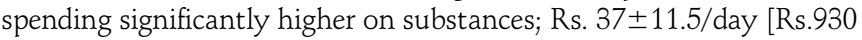
or (US\$13)/month] compared to females. Overall, $41 \%$ of the cost is spent on cigarettes followed by $27 \%$ on local ghutka. The main reasons for using substances were peer pressure, easy availability of substances, stress, liking of taste and to treat toothache.

Conclusion This study concluded that use of substances is higher among young males and they are spending substantial amount on those substances. To prevent this population, regular awareness campaigns may be held at community and school level so that, continuous re-enforcement make them to quit from using any substances.

\section{P2-464 INCIDENCE AND MAIN CAUSES OF SEVERE MATERNAL MORBIDITY IN SÃO LUÍS/MARANHÃO-BRAZIL: A LONGITUDINAL STUDY}

doi:10.1136/jech.2011.142976l.92

\begin{abstract}
${ }^{1}$ A P Moraes, ${ }^{1,2} \mathrm{~S}$ Barreto, ${ }^{1,2} \mathrm{~V}$ Passos, ${ }^{*}{ }^{3} \mathrm{P}$ Golino, ${ }^{4} \mathrm{~J}$ Costa, ${ }^{4} \mathrm{M}$ Vasconcelos. ${ }^{1}$ Public Health Postgraduate Program, Faculty of Medicine, Federal University of Minas Gerais, Belo Horizonte, Minas Gerais, Brazil; ${ }^{2}$ Faculty of Medicine, Federal University of Minas Gerais, Belo Horizonte, Minas Gerais, Brazil; ${ }^{3}$ Maternity Marly Sarney, São Luís, Maranhão, Brazil; ${ }^{4}$ Gynecology and Obstetrics Residence, Federal University of Maranhão, São Luís, Maranhão, Brazil
\end{abstract}

Introduction The evaluation of severe maternal morbidity has been used in the monitoring of maternal health. The objective of this study is to estimate its incidence and main causes in São LuísMaranhão, one of the poorest cities in Brazil.

Methods A prospective longitudinal study, carried out in two public high risk maternity clinics and two intensive care units (ICUs) of reference to obstetrical cases of the city. During the period of 1 March 2009 and 28 February 2010, all cases of severe maternal morbidity were identified following Mantel's and Waterstone's criteria. Socio-demographic characteristics and healthcare data of the extremely severe cases were compared to the less severe cases.
The Fisher, $\chi^{2}$, Student $t$, and Mann-Whitney tests were used for a statistical analysis, with significance levels of $<0.05$.

Results 127 cases of severe maternal morbidity among 8493 deliveries were identified, leading to an incidence of 15.0/1000 deliveries. 122 women were interviewed, five cases were lost (3.9\%). 121 cases fell under Waterstone's criteria and 29 under Mantel's criteria, corresponding to incidences of $14.1 / 1000$ and 3.4/1000 deliveries, respectively. These rates are lower than those described in the literature, possibly due to case loss. The main causes of morbidity were hypertension during pregnancy, more frequent in less severe cases $(p=0.001)$ and obstetrical haemorrhage, more common among extreme severe cases $(p=0.01)$.

Conclusion The obstetrical disorders are the main causes of severe maternal morbidity in São Luís/Maranhão. The investigation and monitoring of severe morbidity can contribute to improve the obstetrical assistance in the city.

\section{P2-465 REGIONAL VARIATION IN HISTOPATHOLOGY-SPECIFIC INCIDENCE OF INVASIVE CERVICAL CANCER AMONG PERUVIAN WOMEN}

doi:10.1136/jech.2011.142976l.93

${ }^{1} \mathrm{C}$ P Campbell, ${ }^{* 2} \mathrm{M}$ P Curado, ${ }^{1} \mathrm{~S}$ Harlow, ${ }^{1} \mathrm{~A}$ Soliman. ${ }^{1}$ Department of Epidemiology, School of Public Health, University of Michigan, Ann Arbor, Michigan, USA; ${ }^{2}$ Cancer Information Section, International Agency for Research on Cancer, Lyon, France

Introduction This study aimed to evaluate cervical cancer patterns in Peru by examining the variation in two common histopathologic types, squamous cell carcinoma (SCC) and adenocarcinoma (ADC), and analysing differences over time.

Methods Data on invasive cervical cancer incidence was obtained from three population-based cancer registries in Peru: Lima, Arequipa, and Trujillo. A cervical cancer-specific quality assessment was performed on each registry. Crude and age-specific incidence rates per 100000 were calculated for overall, SCC- and ADC-specific cervical cancers, and time trends analysed.

Results Lima and Trujillo demonstrated acceptable data quality; however, Arequipa was questionable. Incidence rates for overall cervical cancer were significantly different across registries: Arequipa (47.2), Trujillo (36.1), and Lima (18.9). Rates for SCC were significantly lower in Lima (14.0) as compared to Arequipa (29.7) and Trujillo (30.0). Rates for ADC did not differ significantly across registries. Time trend analyses showed significant declines in overall and SCC-specific rates in Trujillo. No other time trends were found. Age-specific analyses showed that young women (15-29 years) in Trujillo and Arequipa experienced significant increases in ADCspecific rates over time.

Conclusion Cancer registry data showed that overall and histopathology-specific cervical cancer incidence rates varied across regions of Peru, and over time. The use of cancer registry data proved to be an efficient method for evaluating cervical cancer incidence patterns in Peru. We suggest supplementing current screening methods with newer preventive methods to combat the rising incidence of $\mathrm{ADC}$ among young women in Peru.

\section{P2-466 AIR POLLUTION EFFECTS IN RESPIRATORY HEALTH IN SICK POPULATION: A MULTILEVEL STUDY IN BUCARAMANGA COLOMBIA}

doi:10.1136/jech.2011.142976l.94

1,2 L A Rodriguez, ${ }^{3} \mathrm{H}$ Castro, ${ }^{2} \mathrm{~J} J$ Rey. ${ }^{1}$ Universidad Industrial de Santander, Bucaramanga, Santander, Colombia; ${ }^{2}$ Observatorio de Salud Pública de Santander, 